\title{
Treino de estimulação de memória e a funcionalidade do idoso sem comprometimento cognitivo: uma revisão integrativa
}

\author{
Memory stimulation training and the functionality of the elderly \\ without cognitive impairment: an integrative review
}

Erika Carla Cavalcanti Gomes (https://orcid.org/0000-0003-3156-7053) ${ }^{1}$

Sandra Lopes de Souza (https://orcid.org/0000-0001-5695-7344) ${ }^{1}$

Ana Paula de Oliveira Marques (https://orcid.org/0000-0003-0731-8065) ${ }^{1}$

Marcia Carrera Campos Leal (https://orcid.org/0000-0002-3032-7253) ${ }^{1}$

${ }^{1}$ Centro de Ciências da

Saúde, Universidade Federal de Pernambuco. Av. da Engenharia, Cidade Universitária. 50670420 Recife PE Brasil. erikacarlagomes@ yahoo.com.br

\begin{abstract}
The scope of this article is to identify the repercussions of the effect of memory stimulation in healthy elderly individuals. It is an integrative review of the literature, namely of articles published in the last seven years in English, Portuguese and Spanish. The selection was performed through cross-checking of key words: health of the elderly, memory and cognition in the Lilacs, Medline and IBECS databases. The inclusion criteria were: original article; addressing key issue; full text electronically available; population 60 years and above without diagnosis of cognitive impairment. Twelve articles were selected and it was identified that the effect of memory stimulation in the elderly without cognitive impairment can improve performance in advanced, instrumental and basic activities of daily living and in psychological, social and quality of life components in the elderly. Based on analysis of the articles it is important to emphasize the need for further longitudinal and intervention studies focused on cognitive stimulation in the elderly without cognitive impairment and that future research can overcome such limitations by working towards efficient monitoring and evaluation, which is more rigorous and committed to facilitate the cognitive performance of the elderly in daily activities.
\end{abstract}

Key words Health of the Elderly, Memory, Cognition
Resumo $O$ objetivo deste artigo é identificar as repercussões do efeito de estimulação de memória em idosos saudáveis. Revisão integrativa da literatura, de artigos publicados nos últimos sete anos, nos idiomas inglês, português e espanhol. A seleção foi realizada por meio de cruzamentos dos descritores: saúde do idoso, memória e cognição, nas bases de Lilacs, Medline e IBECS. Os critérios de inclusão foram: ser artigo original; responder a questão norteadora; ter disponibilidade eletrônica na forma de texto completo; ter como população de estudos idosos a partir de 60 anos sem diagnóstico de comprometimento cognitivo. Foram selecionados 12 artigos e identificado que o efeito de estimulação de memória em idosos sem comprometimento cognitivo pode melhorar no desempenho nas atividades avançadas, instrumentais e básicas de vida diária, nos componentes psicológicos, sociais e de qualidade de vida dos idosos. De acordo com a análise dos artigos é importante ressaltar a necessidade de mais estudos longitudinais $e$ de intervenção com foco na estimulação cognitiva em idosos sem comprometimento cognitivo e que futuros estudos podem superar tais limitações através de pesquisas na área que visem um bom monitoramento e avaliações mais rigorosas, empenhadas em facilitar o desempenho cognitivo dos idosos nas atividades cotidianas.

Palavras-chave Saúde do Idoso, Memória, Cognição 


\section{Introdução}

O crescimento populacional é uma realidade, especialmente tratando-se da população idosa, cada vez mais evidente nos países em desenvolvimento, a exemplo do Brasil, cuja expectativa para os próximos 20 anos poderá chegar a aproximadamente 30 milhões de idosos, ou seja, 13\% da população $0^{1}$. Essa realidade também é um reflexo dos avanços da medicina moderna, da redução da mortalidade e do aumento da expectativa de vida. No entanto, tais aspectos podem alertar quanto à incidência de afecções e distúrbios advindos da idade, principalmente doenças crônicas degenerativas que irão repercutir diretamente na capacidade funcional do idoso ${ }^{2}$.

Dentre as diversas alterações fisiológicas oriundas do processo natural de envelhecimento, a exemplo do processo de atrofia cerebral, dilatação de sulcos e ventrículos e a perda de neurônios, componentes relevantes para a manutenção da capacidade cognitiva, em destaque, a memória, elemento que está intimamente envolvida na funcionalidade e na participação do idoso na sociedade $^{3}$.

A capacidade funcional é um importante indicador para o envelhecimento bem sucedido, porém vale considerar que os conceitos de "desempenho" e "capacidade funcional" são distintos, mas que se complementam: o primeiro avalia as atividades cotidianas que o idoso realiza no seu dia a dia; e o segundo analisa o potencial que o indivíduo possui para executar tais tarefas. É avaliada por meio de instrumentos padronizados que analisam o desempenho dos idosos nas atividades básicas de vida diária (ABVD), que são: tomar banho, se vestir, ir ao banheiro, e atividades instrumentais de vida diária (AIVD), como telefonar, gerir finanças, realizar compras e nas atividades avançadas de vida diária (AAVD), que são tarefas baseadas em condutas intencionais, envolvidas com o funcionamento físico, mental e social e que permitem ao indivíduo desenvolver múltiplos papeis sociais e a manutenção de boa saúde mental e qualidade de vida ${ }^{4,5}$.

Estudos indicam que diversos fatores podem ser determinantes ao envelhecimento cognitivo: eventos biológicos, hábitos de vida e o ambiente no qual o idoso está inserido. Geralmente, tais aspectos favorecem a degeneração psicobiológica característica do processo de envelhecimento e que promove a inatividade e o déficit nas habilidades cognitivas, e assim as queixas de memória relacionadas com as atividades de vida diária ficam mais evidentes no cotidiano dos idosos ${ }^{5}$.
As queixas de memória mais comuns entre a população idosa estão ligadas a dificuldade de armazenar informações recentes, conseguir resgatá-las e manter-se atento nas atividades do cotidiano ${ }^{5}$. Normalmente, são indivíduos que não lembram nomes de pessoas conhecidas, compromissos importantes, objetos pessoais, esquecem onde guardaram documentos e dinheiro, fogo aceso, administração de medicamentos entre outros acontecimentos que estão diretamente relacionados com o seu desempenho no dia a dia e que podem acarretar em dificuldades na manutenção de sua autonomia ${ }^{6}$.

Os prejuízos funcionais decorrentes das queixas de memórias podem acarretar nos idosos a perda da autoestima, sentimento de inutilidade, isolamento social, familiar e desencadear o aparecimento de outras doenças, a exemplo dos transtornos de ansiedade e depressão ${ }^{7}$. Pesquisas científicas sugerem que as falhas advindas da memória e o envelhecimento é um fenômeno inato do processo natural da senescência, apesar de ainda não haver estudos conclusivos sobre o comprometimento cognitivo se tratar de um fato inexorável do envelhecimento ou se é oriundo de um fenômeno multifatorial que resulta de um decréscimo de estímulos sociais, psicológicos e biológicos ${ }^{8,9}$.

Nesse contexto, a evolução do declínio cognitivo seja advinda de um processo fisiológico ou não, as queixas subjetivas sobre o desempenho mnemônico são reais no cotidiano dos idosos e devem ser investigadas e promover procedimentos e estratégias de intervenção capazes de minimizar os déficits do declínio cognitivo e promover ações de prevenção à saúde do idoso ${ }^{10}$.

Sendo assim, o objetivo desta revisão integrativa foi identificar quais as relações entre o treino de estimulação de memória em idosos sem comprometimento cognitivo e sua funcionalidade.

\section{Método}

Trata-se de uma revisão integrativa da literatura, cujo método permite uma investigação criteriosa e sistematizada sobre determinada problemática no campo científico, com o objetivo de identificar eventuais lacunas do conhecimento científico ${ }^{11}$.

Para a construção desta revisão de literatura foram estipuladas as seguintes etapas: estabelecimento da questão norteadora, seleção e obtenção dos artigos (critérios de inclusão e exclusão); avaliação dos estudos pré-selecionados; discussão dos resultados e apresentação da revisão 
integrativa $^{12}$. Na primeira etapa na pesquisa foi estruturada a seguinte questão norteadora: Quais as evidências científicas publicadas nos últimos sete anos que identificaram as relações sobre o efeito do treino de estimulação de memória na funcionalidade do idoso sem comprometimento cognitivo?

A etapa seguinte foi selecionar os artigos, através da busca por publicações da literatura científica, no período de janeiro 2009 a maio de 2017, nos idiomas inglês, português e espanhol, na base de dados da Biblioteca Virtual em Saúde (BVS), na qual é possível realizar buscas simultâneas nas principais bases de dados científicos nacionais e internacionais: Lilacs (Literatura Latino -Americana e do Caribe em Ciências da Saúde), Medline (Literatura Internacional em Ciências da Saúde), IBECS (Índice bibliográfico Espanhol de Ciências da Saúde).

Os critérios de inclusão estabelecidos para a seleção dos artigos foram: ser artigo original; responder a questão norteadora; ter disponibilidade eletrônica na forma de texto completo, publicado no período pré-determinado nos idiomas supracitados; ter como população de estudos idosos sem diagnóstico de comprometimento cognitivo. Foram excluídos: pesquisas que consideraram idosos indivíduos com idade inferior a 60 anos, estudos repetidos em uma ou mais bases de dados.

Para selecionar os artigos foram utilizadas três palavras chaves indexadas nos Descritores em Ciências da Saúde (DeCS): saúde do idoso, memória e cognição. A partir da escolha dos termos mencionados, foram realizadas todas as possibilidades de cruzamentos entre os descritores selecionados, por meio de duas estratégias de busca (A e B). No método A as palavras foram cruzadas utilizando-se o ícone AND; e na estratégia $\mathrm{B}$ através do ícone OR.

A análise crítica dos artigos pré-selecionados foi realizada a partir da aplicação de dois instrumentos: Critical Appraisal Skill Programme (CASP) e Agency for Healthcare and Research and Quality (AHRQ). A utilização das avaliações supracitadas teve o objetivo de analisar a qualidade metodológica dos estudos incluídos nesta revisão integrativa ${ }^{11,12}$.

A CASP é um instrumento que classifica os artigos de acordo com pontuações que variam de seis a dez (boa qualidade metodológica e viés reduzido e cinco pontos ou menos (qualidade metodológica satisfatória, mas com risco considerável. Nesta revisão foram considerados os artigos classificados entre 6 a 10 pontos $^{12}$.
O AHRQ é uma avaliação que destaca dos estudos em seis níveis de evidência: (1) revisão sistemática ou metanálise; (2) ensaios clínicos randomizados; (3) ensaios clínicos sem randomização; (4) estudos de coorte e de caso-controle; (5) revisão sistemática de estudos descritivos e qualitativos e (6) único estudo descritivo ou qualitativo $^{12}$.

As avaliações supracitadas contemplam em sua análise: a identificação do artigo original, as características metodológicas do estudo e a avaliação dos resultados selecionados nesta revisão.

\section{Resultados}

Realizados os possíveis cruzamentos entre os descritores pré-estabelecidos, foram encontrados 360 artigos, que passaram por uma prévia seleção por meio da leitura dos títulos e resumos, quando necessário. Ao término dessa fase foram selecionados 47 artigos e lidos na íntegra com o objetivo de identificar os mesmos aos critérios de inclusão atribuídos a esta revisão (Figura 1).

Após a etapa supracitada, foram excluídos 35 artigos, gerando-se uma amostra final de 12, apresentados no Quadro 1. Na base de dados Medline foram encontrados seis artigos que se enquadram nos critérios de inclusão mencionados anteriormente, destes cinco foram publicados na língua inglesa no ano de 2015. Na Lilacs foram incluídos seis estudos, cinco na língua portuguesa entre os anos 2012 e 2010, e no IBECS não foram encontrados artigos referente ao tema da pesquisa.

No que diz respeito às origens das publicações, foram selecionados sete artigos internacionais em países da América do sul, da Europa e da Ásia. No cenário nacional, foram selecionados cinco artigos publicados nas áreas: geriatria, saúde pública e psicologia. Quanto ao desenho metodológico dos artigos selecionados, verificou-se que 10 utilizaram como método estudos de intervenção e dois utilizaram o delineamento transversal.

De acordo com os artigos selecionados, $42 \%$ estudaram os efeitos da estimulação de memória em relação ao desempenho dos idosos nas atividades avançadas de vida diária e 70\% dos estudos utilizaram o Mini Exame do Estado Mental (MEEM) nas intervenções. Em relação à população estudada em $80 \%$ das pesquisas o percentual de participantes do sexo feminino foi superior a masculina e $20 \%$ dos estudos estudaram especificamente a população de mulheres idosas. 


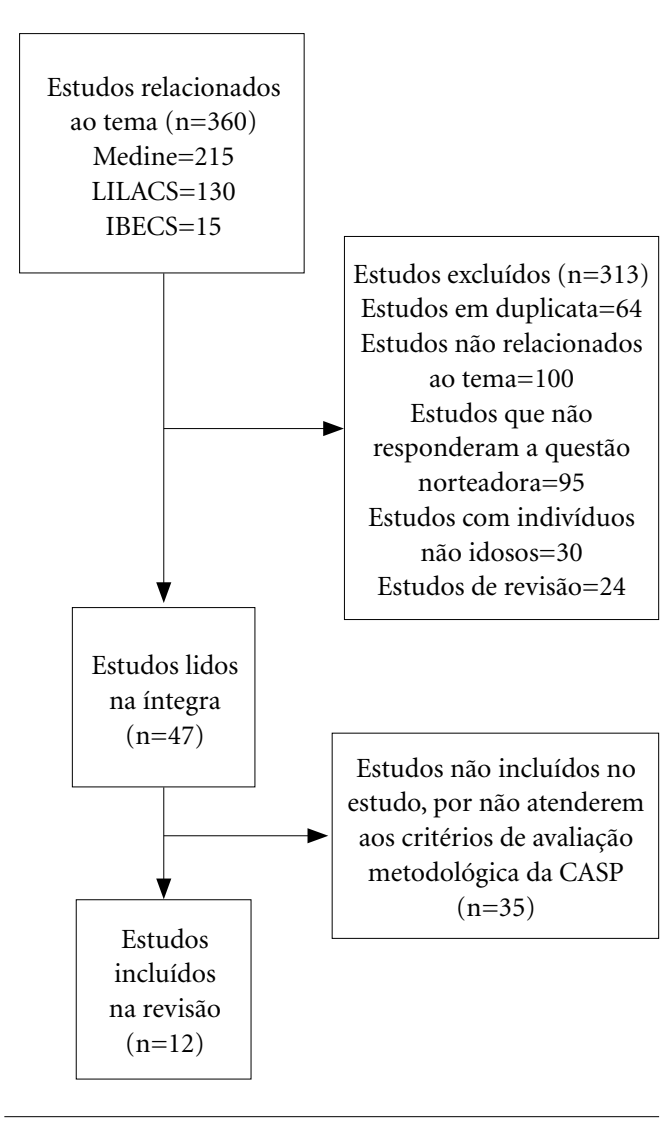

Figura 1. Diagrama dos estudos incluídos e excluídos de acordo com os critérios de inclusão e exclusão estabelecidos para o estudo.

Apenas 25\% dos artigos selecionados não possuíam características de intervenção, mas sim de avaliação e acompanhamento, estes artigos foram incluídos neste estudo pelo fato de considerarem a realização das atividades produtivas e de vida diária como exercícios que estimulam a memória quando comparados a idosos que não realizam ou deixaram de realizar tais tarefas.

Identificou-se também que 50\% dos estudos apontaram que a estimulação cognitiva em idosos sem comprometimento nas habilidades mnemônicas tem efeito positivo em componentes psicológicos, relações sociais e na qualidade de vida e destacam a necessidade de intervenções com atividades voltadas a tarefas que são significativas para a manutenção da autonomia dos idosos.

Todos os artigos selecionados afirmaram que a memória sofre alterações significativas no decorrer do processo de envelhecimento, independente do diagnóstico de comprometimento cognitivo. A memória episódica, operacional, atenção e concentração são componentes que geralmente estão associados aos déficits na codificação e na recuperação de informação nos idoso e enfatizam que o treino cognitivo pode melhorar o desempenho da memória e ajudar na manutenção da funcionalidade do idoso na execução de atividades cotidianas.

Quanto às recomendações mais de $60 \%$ dos estudos revelaram que o treino de estimulação de memória pode ser um fator de proteção na manutenção do declínio cognitivo e da capacidade funcional dos idosos, principalmente no que se refere às atividades avançadas de vida diária, e sugerem que os exercícios para a estimulação de memória têm melhor efeito quando associados com tarefas realizadas no dia a dia.

Os artigos internacionais enfatizaram a necessidade de mais estudos na temática em questão, visto que tais pesquisas podem contribuir para o planejamento e a execução de intervenções direcionadas para a prevenção de doenças relacionadas com o comprometimento cognitivo e, principalmente, comprometidas com a promoção da saúde e qualidade de vida dos idosos.

\section{Discussão}

O envelhecimento populacional é uma realidade nos países desenvolvidos e em desenvolvimento e constitui um desafio para diversos setores da sociedade, destacando-se o da saúde ${ }^{13}$. Tais mudanças do perfil populacional mundial também sugerem alterações no cenário epidemiológico desses indivíduos, principalmente no que se refere a doenças crônico-degenerativas, a exemplo das doenças desencadeadas por declínio cognitivo e alterações de humor ${ }^{14}$.

A equivalência de artigos internacionais e nacionais incluídos nesta pesquisa sinaliza o quanto essa realidade é relevante e que necessita ser estudada com cautela, visto que a percepção do funcionamento cognitivo é considerada um indicador importante na condição de saúde dos idosos, pois podem potencializar morbidades neuropsicológicas, como depressão e ansiedade, e assim interferir na qualidade de vida desses indivíduos.

Estudos sugerem que o treino mnemônico pode estimular alterações no funcionamento cognitivo e promover melhoras da funcionalidade e independência do idoso ${ }^{14-16}$. Tal assertiva pode ser observada de acordo com o quantitativo 
Quadro 1. Apresentação da síntese dos trabalhos incluídos na revisão integrativa.

\begin{tabular}{|c|c|c|c|c|c|}
\hline $\mathbf{N}$ & Artigo/autor & Periódico & $\begin{array}{c}\text { Ano de } \\
\text { publicação }\end{array}$ & Idioma & Principal objetivo \\
\hline 01 & \begin{tabular}{|l|} 
Lifetime Occupation \\
and Late-Life Cognitive \\
Performance Among \\
Women. \\
(Ribeiro PCC, Lorenço RA) \\
\end{tabular} & $\begin{array}{l}\text { Health Care } \\
\text { Women Int; }\end{array}$ & 2015 & Inglês & $\begin{array}{l}\text { Comparar o desempenho } \\
\text { cognitivo de idosas que } \\
\text { trabalhavam como donas de } \\
\text { casa e àquelas que trabalhavam } \\
\text { regularmente remuneradas. }\end{array}$ \\
\hline 02 & $\begin{array}{l}\text { Efficacy of memory } \\
\text { training in healthy } \\
\text { community-dwelling } \\
\text { older people: study } \\
\text { protocol for a } \\
\text { randomized controlled } \\
\text { trial. } \\
\text { (Perez A. et al. }) \\
\end{array}$ & BMC Geriatra; & 2015 & Inglês & $\begin{array}{l}\text { Avaliar a eficácia de oficinas de } \\
\text { memória nas habilidades de } \\
\text { autopercepção, função executiva } \\
\text { e qualidade de vida em idosos } \\
\text { saudáveis. }\end{array}$ \\
\hline 03 & $\begin{array}{l}\text { Cognitive performance } \\
\text { and engagement in } \\
\text { physical, social and } \\
\text { intellectual activities in } \\
\text { older adults: The FIBRA } \\
\text { study } \\
\text { (Sposito G, Yassuda MS) }\end{array}$ & $\begin{array}{l}\text { Dement. } \\
\text { neuropsychol; }\end{array}$ & 2015 & Inglês & $\begin{array}{l}\text { Investigar a relação entre } \\
\text { o engajamento de idosos } \\
\text { sem diagnóstico de } \\
\text { comprometimentos cognitivos } \\
\text { nas atividades avançadas de vida } \\
\text { diária e domínios da cognição } \\
\text { em idosos residentes em sete } \\
\text { localidades brasileiras. }\end{array}$ \\
\hline 04 & $\begin{array}{l}\text { Advance Activities of } \\
\text { daily living and incidence } \\
\text { of decline in the elderly } \\
\text { the SABE study. } \\
\text { (Dias EG et al.) }\end{array}$ & $\begin{array}{l}\text { Cad Saude } \\
\text { Publica; }\end{array}$ & 2015 & Português & $\begin{array}{l}\text { Avaliar o impacto das atividades } \\
\text { avançadas de vida diária na } \\
\text { incidência de declínio cognitivo } \\
\text { em idosos sem déficit cognitivo. }\end{array}$ \\
\hline 05 & $\begin{array}{l}\text { Interventions for older } \\
\text { persons reporting } \\
\text { memory difficulties: a } \\
\text { randomized controlled } \\
\text { pilot study. (Cohen- } \\
\text { Mansfield J. et al) }\end{array}$ & $\begin{array}{l}\text { Int J Geriatr } \\
\text { Psychiatry }\end{array}$ & 2015 & Inglês & $\begin{array}{l}\text { Comparar três intervenções } \\
\text { diferentes para pessoas que } \\
\text { relatam dificuldades de memória: } \\
\text { promoção da saúde, formação } \\
\text { cognitiva e um curso centrado na } \\
\text { participação }\end{array}$ \\
\hline 06 & $\begin{array}{l}\text { Memory banking: a life } \\
\text { story intervention for } \\
\text { aging preparation and } \\
\text { mental health promotion. } \\
\text { (Zanjani F, Downer BG, } \\
\text { Hosier AF, Watkins JD) } \\
\end{array}$ & J Aging Health; & 2015 & Inglês & $\begin{array}{l}\text { O objetivo deste estudo é analisar } \\
\text { a viabilidade de Bancos de } \\
\text { memória e determinar o impacto } \\
\text { da intervenção na saúde mental, } \\
\text { apoio social e qualidade de vida } \\
\text { em mulheres idosas }\end{array}$ \\
\hline 07 & \begin{tabular}{|l|} 
Treino cognitivo em \\
adultos maduros e idosos: \\
impacto de estratégias \\
segundo faixas de \\
escolaridade \\
(Teixeira-Fabrício A. et al.)
\end{tabular} & $\begin{array}{l}\text { Psico USF } \\
\text { (Impr.); }\end{array}$ & 2012 & Português & $\begin{array}{l}\text { Testar a eficácia de treino } \\
\text { cognitivo de seis sessões, baseado } \\
\text { na apresentação e prática } \\
\text { de estratégias de memória } \\
\text { (categorização) e na realização } \\
\text { de atividades que recrutam as } \\
\text { funções executivas, oferecido a } \\
\text { idosos saudáveis. }\end{array}$ \\
\hline 08 & $\begin{array}{l}\text { Desempenho cognitivo } \\
\text { em diferentes níveis de } \\
\text { escolaridade de adultos e } \\
\text { idosos ativos } \\
\text { (Coelho FM. et al.) }\end{array}$ & $\begin{array}{l}\text { Rev. bras. } \\
\text { geriatra. } \\
\text { gerontol. } \\
\text { (Impr.); }\end{array}$ & 2012 & Português & $\begin{array}{l}\text { Comparar o desempenho } \\
\text { cognitivo em diferentes níveis } \\
\text { de escolaridade de indivíduos } \\
\text { adultos e idosos praticantes de } \\
\text { atividade física. }\end{array}$ \\
\hline
\end{tabular}


Quadro 1. Apresentação da síntese dos trabalhos incluídos na revisão integrativa.

\begin{tabular}{|c|c|c|c|c|c|}
\hline $\mathbf{N}$ & Artigo/autor & Periódico & $\begin{array}{c}\text { Ano de } \\
\text { publicação }\end{array}$ & Idioma & Principal objetivo \\
\hline 09 & $\begin{array}{l}\text { Efeitos de um treino } \\
\text { de atenção, memória } \\
\text { e funções executivas } \\
\text { na cognição de idosos } \\
\text { saudáveis } \\
\text { (Irigaray TQ, Filho IG, } \\
\text { Schneider RH) }\end{array}$ & $\begin{array}{l}\text { Psicol. (Univ. } \\
\text { Fed. Rio Gd. Sul, } \\
\text { Impr.); }\end{array}$ & 2012 & Português & $\begin{array}{l}\text { Verificar os efeitos de um treino } \\
\text { de atenção, memória e funções } \\
\text { executivas na cognição de idosos } \\
\text { saudáveis. }\end{array}$ \\
\hline 10 & $\begin{array}{l}\text { Treino cognitivo } \\
\text { para idosos baseado } \\
\text { em estratégias de } \\
\text { categorização e cálculos } \\
\text { semelhantes a tarefas do } \\
\text { cotidiano } \\
(\text { Silva } T B L) \\
\end{array}$ & $\begin{array}{l}\text { Rev. bras. } \\
\text { geriatr. gerontol. } \\
\text { (Impr.); }\end{array}$ & 2011 & Português & $\begin{array}{l}\text { Testar a eficácia de um programa } \\
\text { de treino cognitivo baseado } \\
\text { em tarefas ecológicas, que } \\
\text { mimetizam tarefas de compra, } \\
\text { envolvendo a memorização de } \\
\text { itens de supermercado e cálculos } \\
\text { matemáticos simples. }\end{array}$ \\
\hline 11 & $\begin{array}{l}\text { Efeitos de um treino } \\
\text { cognitivo na qualidade } \\
\text { de vida e no bem-estar } \\
\text { psicológico de idosos } \\
\text { (Irigaray TQ, Schneider } \\
\text { RH, Gomes I) }\end{array}$ & $\begin{array}{l}\text { Psicol. (Univ. } \\
\text { Fed. Rio Gd. } \\
\text { Sul, Impr.); Cad } \\
\text { Saude Publica; }\end{array}$ & 2011 & Português & $\begin{array}{l}\text { Verificar os efeitos de um treino } \\
\text { cognitivo na qualidade de vida } \\
\text { e no bem-estar psicológico de } \\
\text { idosos. }\end{array}$ \\
\hline 12 & $\begin{array}{l}\text { Treino de memória } \\
\text { episódica com ênfase em } \\
\text { categorização para idosos } \\
\text { sem demência e depressão } \\
\text { (Carvalho FCR, Neri } A L, \\
\text { Yassuda MS) }\end{array}$ & $\begin{array}{l}\text { Psicol. (Univ. } \\
\text { Fed. Rio Gd. } \\
\text { Sul, Impr.); Cad } \\
\text { Saude Publica; }\end{array}$ & 2010 & Português & $\begin{array}{l}\text { Verificar os efeitos do treino de } \\
\text { memória episódica com idosos } \\
\text { saudáveis brasileiros }\end{array}$ \\
\hline
\end{tabular}

encontrado de artigos selecionados para este estudo que utilizaram a intervenção do tipo casocontrole na metodologia, e que possibilita testar hipóteses de causalidade entre efeito da estimulação cognitiva e a funcionalidade do idoso. Fato que justifica a maioria dos artigos apresentarem nível de evidência superior a seis, de acordo com a análise metodológica.

No entanto, estudos de treino cognitivo no Brasil ainda são escassos, não havendo dados suficientes para confirmar se os achados nas publicações internacionais podem ser comparados à nossa população, considerando que as características sociodemográficas e epidemiológicas são diferentes ${ }^{5}$. Neste estudo foi possível identificar que o número de publicações internacionais recentes é superior às pesquisas realizadas no âmbito nacional.

Outra questão importante que foi ressaltada nos artigos 01,02, 04, 06, 07, 09 e 10 (Quadro 1) é a relação entre o declínio cognitivo e as perdas funcionais, principalmente na execução das atividades avançadas de vida diária (AAVD) seguidas pelas perdas nas atividades instrumentais de vida diária (AIVD) e atividades básicas de vida diária $(\mathrm{ABVD})^{17}$. Os estudos supracitados sugerem que alterações no desempenho das AAVD podem representar primeiros sinais de comprometimentos cognitivos leves que podem gerar dificuldades no desempenho de habilidades para manter o trabalho, viajar e planejar viagens, participação em grupos ou movimentos comunitários, dirigir, planejar eventos ou jogar.

As AAVD estão ligadas com atividades volitivas e são influenciadas por contextos sociais, culturais e fatores motivacionais e essas questões irão refletir em uma vida comunitária independente $^{17,18}$. O artigo 01 selecionado para esta revisão faz uma reflexão sobre essa assertiva, que a manutenção de atividades profissionais e não profissionais no envelhecimento pode estar associada ao melhor funcionamento cognitivo.

Os artigos selecionados indicaram a predominância do sexo feminino em suas amostras. Não existe na literatura científica um consenso sobre tal questão, mas sabe-se que as mulheres 
idosas, geralmente, têm uma expectativa maior que a dos homens e culturalmente estão mais envolvidas nas atividades de controle e manutenção domiciliar e que, por isso, têm uma melhor percepção da relação entre as falhas de memória e a capacidade funcional ${ }^{18}$.

No entanto, os artigos internacionais reforçam a importância do ambiente como fonte de experiência cerebral que podem estimular na formação e manutenção de reservas cognitivas ${ }^{19}$. Desse modo, fatores ligados à escolaridade, atividades laborais, lazer vivenciadas ao logo da vida, poderiam favorecer o crescimento neuronal $\mathrm{e}$ neuroplasticidade e assim postergar o aparecimento de déficits cognitivos.

Pesquisas sinalizam que os padrões de atividade e as redes neurais são construídos no início da vida e podem ter um papel determinante para manter a saúde cognitiva durante o processo de envelhecimento ${ }^{19-21}$. Os estudos de treino de memória selecionados nesta revisão acreditam que os idosos têm capacidade de aprender e empregar estratégias mnemônicas e que os efeitos advindos dessas estimulações podem ser usados nas atividades cotidianas e que as repercussões positivas podem ser mantidas em até seis meses.

Desse modo, a literatura científica a respeito do treino de memória confirma a noção da plasticidade da memória no envelhecimento, mas sugere limitação na generalização e manutenção dos efeitos desses programas, e enfatiza a importância no planejamento de atividades que sejam significativas nas tarefas cotidianas dos idosos ${ }^{22,23}$.

Neste estudo de revisão observou-se que os programas de treino de estimulação cognitiva diferem em diversos aspectos, a exemplo da duração, estratégias e metodologias, e por isso são encontradas diversas repercussões relacionadas aos seus efeitos e quanto à manutenção em longo prazo. No entanto, existe um consenso entre os estudos selecionados que os treinos têm o objetivo de maximizar as funções cognitivas e prevenir futuros declínios cognitivos.

Nos estudos internacionais é possível perceber um quantitativo significativo de pesquisas sobre treino cognitivo em idosos sem diagnóstico de demência e/ou depressão ${ }^{24-26}$. Todos os artigos internacionais selecionados apontaram para a importância de se investir em mais estudos de intervenção com o objetivo de realizar mais programas voltados à prevenção do aparecimento de declínio cognitivo e principalmente, de promoção da saúde do idoso.

No Brasil, os artigos científicos sobre memória com foco em idosos sem comprometimento cognitivo ainda são considerados temas pouco estudados, principalmente tratando-se em estudos longitudinais e de intervenção ${ }^{27,28}$. O presente estudo observou que os trabalhos nacionais devem ter um maior investimento em testar a eficácia de programas de reabilitação cognitiva para a população em questão e comprometidos com os desafios cognitivos diários dos idosos.

No envelhecimento saudável existe a possibilidade de compensação dos déficits cognitivos ${ }^{29,30}$. As pesquisas selecionadas para esta revisão indicaram que o idoso saudável é capaz de aproximar se desempenho atual com sua capacidade máxima, por meio da plasticidade cognitiva.

De um modo geral objetivo de uma revisão integrativa consiste em combinar estudos de diversas metodologias, áreas de conhecimento e permite combinar informações que ajudam a identificar possíveis lacunas nos estudos e consequentemente, nas intervenções clínicas. No entanto, se ressalta que os meios de divulgação das pesquisas ainda são limitados devido às exigências dos periódicos científicos, sendo a necessidade de outros idiomas e de recursos financeiros para obtenção das informações fatores limitantes para a elaboração desta revisão.

\section{Conclusão}

A partir do objetivo desta revisão integrativa foi possível identificar as relações sobre efeito do treino de estimulação cognitiva em idosos saudáveis e sua funcionalidade, destacando-se que tal intervenção pode favorecer a melhora no desempenho nas AAVD, AIVD, ABVD, e na possibilidade de favorecer o desempenho atual com sua capacidade funcional máxima, e consequentemente, na promoção da qualidade de vida do idoso.

No que diz respeito às lacunas no conhecimento encontradas sobre a temática estudada, sugere-se a necessidade de mais investimentos em estudos longitudinais, de intervenção, comprometidos em incentivar a realização de programas de estimulação cognitiva em idosos, sobretudo saudáveis, capazes de contribuir para um melhor monitoramento da capacidade funcional e na prevenção de doenças neurodegenerativas, $\mathrm{e}$ assim, favorecer a autonomia e a independência dos idosos.

Em síntese, deve-se ressaltar a importância do tema referente às informações supracitadas para o campo da Neurociência, Geriatria e Gerontologia, visto que o bom funcionamento cognitivo 
está diretamente interligado com a capacidade funcional do idoso. Futuras pesquisas podem superar as limitações e as lacunas apresentadas neste estudo com inclusão de estudos com monitoramento e avaliações mais rigorosas e empenhadas em facilitar e aprimorar o desempenho nas atividades cotidianas.

\section{Colaboradores}

ECC Gomes - Trabalhou na concepção, análise dos dados e na redação do artigo; SL Souza e MCC Leal - Trabalharam na revisão crítica do artigo; APO Marques - Trabalhou na redação do artigo e revisão crítica. 


\section{Referências}

1. López Perez-Dias AGL, Calero MD, Navarro-González E. Prediction of cognitive impairment in the elderly by analyzing their performance in verbal fluency and in sustained attention. Rev Neurol 2013; 56(1):17.

2. Willis SL, Tennstedt SL, Marsiske M, Ball K, Elias J, Koepke KM, Morris JN, Rebok JW, Unverzagt FW, Stoddard AM, Wright E, ACTIVE Study Group. For the ACTIVE Study Group, Long-term effects of cognitive training on everyday functional outcomes in older adults. JAMA 2006; 296(23):2805-2814.

3. Jonaitis E, La Rue A, Mueller KD, Koscik RL, Hermann B, Sager MA. Cognitive activities and cognitive performance in middle-aged adults at risk for Alzheimer's disease. Psychol Aging 2013; 28(4):1004-1014

4. Carvalho FCR, Neri AL, Yassuda MS. Treino de memória episódica com ênfase em categorização para idosos sem demência e depressão. Psicologia Reflexão e Crítica 2010; 23(2):317-323.

5. Yassuda MS, Tavares SS, Fortes AG, Neri AL. Treino de memória em idosos saudáveis: Mecanismos e benefícios. Psicologia, Reflexão e Crítica 2006; 19(3):470-481.

6. Aarsten MJ, Smits CH, Van Tiburg T, Knipscheer KCP, Deeg JH. Activity in older adults: cause or consequence of cognitive functionning? A longitudinal study on everyday activities and cognitive performance in older adults. The Journals of Gerontology 2003; 57 B(2):153162.

7. Lasca VB. Treinamento de memória no envelhecimento normal: um programa aplicado a idosos [tese]. Campinas: Universidade Estadual de Campinas; 2003.

8. Salmazo-Silva H, Yassuda M. S. Memory training for older adults with low education: mental images versus categorization. Educational Gerontology 2009; 35(10):890-905

9. Castro-Costa E, Dewey ME, Uchôa E, Firmo JO, Lima-Costa MF, Stewart R. Trajectories of cognitive decline over 10 years in Brazilian elderly population: the Bambuí Cohort Study of Aging. Cad Saude Publica 2011; 27(Supl. 3):S345-350.

10. Argimon IIL, Stein LM. Habilidades cognitivas em indivíduos muito idosos: um estudo longitudinal. Cad Saude Publica 2005; 21(1):64-72.

11. Mendes KDS, Silveira RCCP, Galvão CM. Revisão Integrativa: método de pesquisa para incorporação de evidências na saúde e na Enfermagem. Texto Contexto Enferm 2008; 17(Supl. 4):758-764

12. Souza MT, Silva MD, Carvalho R. Revisão integrativa: o que é e como fazer. Einstein 2010; 8(Supl. 1):102106.

13. Vancarenghi RV, Santos SSC, Barlen ELD, Pelzer MT, Gomes GC, Lange C. Alterações na funcionalidade/ cognição e depressão em idosos institucionalizados que sofreram quedas. Acta paul enferm 2011; 24(Supl. 6):828-833.

14. D’Orsi E, Xavier AJ, Ramos LR. Trabalho, suporte social e lazer protegem idosos da perda funcional: Estudo Epidoso. Rev Saude Publica 2011; 45(4):685-692.
15. Diniz BS, Nunes PV, Yassuda MS, Pereira FS, Flaks MK, Viola LF, Radanovic M, Abreu ID, Borelli DT, Gattaz WF, Forlenza OV. Mild cognitive impairment: cognitive screening or neuropsychological assessment? Rev Bras Psiquiatr 2008; 30(4):316-321.

16. Ramos LR. Fatores determinantes do envelhecimento saudável em idosos residentes em centro urbano: Projeto Epidoso. Cad Saude Publica 2003; 19(3):793-797.

17. Glei DA, Landau DA, Goldman N, Chuang Y, Rodrí guez G, Weinstein M. Participating in social activities helps preserve cognitive function: an analysis of a longitudinal, population-based study of the elderly. Int J Epidemiol 2005; 34(4):864-871.

18. Barnes DE, Cauley JE, Lui L-Y, Fink HA, McCulloch C, Stone KL, Yaffe K. Women who maintain optimal cognitive function into old age. J Am Geriatr Soc 2007; 55(2):259-264

19. Adam S, Bonsang E, Grotz C, Perelman S. Occupational activity and cognitive reserve: implications in terms of prevention of cognitive aging and Alzheimer's disease. Clin Interv Aging 2013; 8:377-390.

20. Akbaraly TN, Portet F, Fustinoni S, Dartigues JF, Artero S, Rouaud O, Touchon J, Ritchie K, Berr C. Leisure activities and the risk of dementia in the elderly: results from the Three-City Study. Neurology 2009; 73(11):854-861.

21. Archat H, Caramelli P, Sameshima K, Nitrini R. Declínio da capacidade cognitiva durante o envelhecimento. Revista Brasileira de Psiquiatria 2005; 27(12):79-82

22. Barulli D, Stern Y. Efficiency, capacity, compensation, maintenance, plasticity: emerging concepts in cognitive reserve. Trends Cogn Sci 2013; 17(10):502-509.

23. De Vriendt P, Gorus E, Cornelis E, Velghe A, Petrovic $\mathrm{M}$, Mets $\mathrm{T}$. The process of decline in advanced activities of daily living: a qualitative explorative study in mild cognitive impairment. Int Psychogeriatr 2012; 24(6):974-986.

24. Vriendt P, Gorus E, Cornelis E, Velghe A, Petrovic M, Mets T. The advanced activities of daily living: a tool allowing the evaluation of subtle functional decline in mild cognitive impairment. J Nutr Health Aging 2013; 17(1):64-71.

25. Guerreiro TC, Veras R, Motta LB. Queixa de memória e disfunção objetiva de memória em idosos que ingressam na Oficina da Memória na UnATI/UERJ. Rev. Bras. Geriatr. Gerontol 2006; 9(1):7-20.

26. Argimon IIL, Stein LM, Xavier FMF, Trentini CM. O impacto de atividades de lazer no desenvolvimento cognitivo de idosos. Revista Brasileira de Ciências do Envelhecimento Humano 2004; 1(1):38-47.

27. Souza JN, Chaves EC. O efeito do exercício de estimulação da memória em idosos saudáveis. Revista da Escola de Enfermagem da USP 2006; 39(10):13-19.

28. Garcia EL, Banegas JR, Pérez-Regadera AG. Social network and health-related quality of life in older adults: A population-based study in Spain. Quality of Life Research 2005; 14(2):511-520. 
29. Carvalho FCR, Neri AL, Yassuda MS. Treino de memória episódica com ênfase em categorização para idosos sem demência e depressão. Psicol. Reflex. Crít 2010; 23(2):317-332.

30. Almeida MHM, Berger, M.L.M, Watanabe, H.A.W. Oficina de memória para idosos: estratégia para promoção da saúde. Interface (Botucatu) 2007; 11(22):271-280.

Artigo apresentado em 12/03/2018

Aprovado em 26/11/2018

Versão final apresentada em 28/11/2018 